\title{
Combined Metabolomics and Network Toxicology to Explore the Molecular Mechanism of Phytolacca acinose Roxb-Induced Hepatotoxicity in Zebrafish Larvae in Vivo
}

\author{
Dan Cao ${ }^{1}$, ${ }^{1,2}$ Chongjun Zhao, ${ }^{1,2}$ Zhiqi Li, ${ }^{1,2}$ Qiqi Fan, ${ }^{1,2}$ Meilin Chen, ${ }^{1}$ Yangyu Jiang, \\ Haiyan Wang, ${ }^{1}$ Hanjun Ning, ${ }^{3}$ Ruichao Lin $\mathbb{D D}^{1,2}$ and Jian Li ${ }^{1}$ \\ ${ }^{1}$ Beijing University of Chinese Medicine, Beijing 102488, China \\ ${ }^{2}$ Beijing Key Lab for Quality Evaluation of Chinese Materia Medica, Beijing 102488, China \\ ${ }^{3}$ Oncology Surgery Department, She Xian Hosipital, Handan 056400, China
}

Correspondence should be addressed to Ruichao Lin; linrch307@sina.com and Jian Li; lijiancn922@126.com

Received 11 August 2021; Revised 17 October 2021; Accepted 21 October 2021; Published 28 November 2021

Academic Editor: Youhua Wang

Copyright $\odot 2021$ Dan Cao et al. This is an open access article distributed under the Creative Commons Attribution License, which permits unrestricted use, distribution, and reproduction in any medium, provided the original work is properly cited.

Phytolacca acinosa Roxb (PAR), a traditional Chinese medicine, has been widely used as a diuretic drug for a long period of time for the treatment edema, swelling, and sores. However, it has been reported that PAR might induce hepatotoxicity, while the mechanisms of its toxic effect are still unclear. In this study, network toxicology and metabolomic technique were applied to explore PAR-induced hepatotoxicity on zebrafish larvae. We evaluated the effect of PAR on the ultrastructure and the function of the liver, predictive targets, and pathways in network toxicology, apoptosis of liver cells by PCR and western blot, and metabolic profile by GC-MS. PAR causes liver injury, abnormal liver function, and apoptosis in zebrafish. The level of arachidonic acid in endogenous metabolites treated with PAR was significantly increased, leading to oxidative stress in vivo. Excessive ROS further activated the p53 signal pathway and caspase family, which were obtained from KEGG enrichment analysis of network toxicology. The gene levels of caspase-3, caspase- 8 , and caspase- 9 were significantly increased by RT-PCR, and the level of Caps 3 protein was also significantly up-regulated through western blot. PAR exposure results in the liver function abnormal amino acid metabolism disturbance and motivates hepatocyte apoptosis, furthermore leading to liver injury.

\section{Introduction}

Phytolacca acinosa Roxb (PAR), namely, Shang $L u$ in Chinese, has a long history in China, which is widely used as a diuretic drug in treating various diseases such as edema, nephritis, ascites, swelling, and sores [1]. PAR is recorded in Chinese Pharmacopoeia and is famous for its multiple functions, such as antibacterial, antiinflammatory, antiviral, antitumor, and for enhancing immunity by modern pharmacological study [2]. However, the toxic performance of PAR cannot be ignored, which may have a significant effect on target organ damage [3]. A study showed that a large dose of esculentoside A, a main active ingredient in PAR, has toxicity on HK-2 (human renal tubular epithelial cells) and the mechanisms of toxicity are associated with cellular oxidative damage and cell apoptosis [4]. Another study found that esculentoside A reduced the viability of HL7702 (human normal liver cells), causing hepatotoxicity [5]. However, until now, there have been few literatures on hepatotoxicity of PAR in vivo, and the mechanism of liver injury is still unclear.

Recently, zebrafish has been used to establish various disease models for high-throughput drug activity and toxicity screening [6-8], especially for a study on drug effects of the liver structure and function $[9,10]$. At the molecular level, its hepatocellular function $[11,12]$ and histopathological changes in a variety of hepatic diseases are much similar to those of humans; therefore, zebrafish is an ideal experimental model for evaluating hepatotoxicity and metabolic research. 
Metabonomic techniques could monitor the dynamic spectrum of endogenous metabolites [13, 14], quickly screen biomarkers with hepatotoxicity conditions, especially appropriate for the multipathway and multitarget toxicity complex system of TCM. Hepatotoxic drugs destroyed the homeostasis of endogenous substances by damaging the cell structure and functions. In addition, metabolomics is a considerable tool for tracing general metabolic changes in biological processes and investigating interactions of drug toxicity with biosomes.

Network toxicology belongs to the branch of the broad category of network pharmacology. By constructing a network model of interaction between toxicity-toxic component-toxic target-effect pathways and analyzing the correlation between specific components in the network toxicology [15] is extremely helpful to explore the toxicity mechanism on TCM.

In this study, for the sake of further clarification of the toxicity on PAR, we used zebrafish (Danio Rerio), a powerful vertebrate model organism, combined with comprehensive analysis of the function, morphology, and operation of hepatotoxicity, as well as metabonomics and network toxicology methods to explore the effects of zebrafish on liver injury and its potential toxicity mechanism.

\section{Materials and Methods}

2.1. Reagents and Materials. Methoxyamine $\mathrm{HCl}$, fatty acid methyl ester (C7-C30, FAMEs) standards, pyridine, and anhydrous sodium sulfate were obtained from SigmaAldrich (St. Louis, MO, USA). MSTFA (N-methyl-N(trimethylsilyl)trifluoroacetamide) with $1 \%$ (vol/vol) trimethylchlorosilane (MSTFA, with 1\% TMCS), methanol (Optima LC-MS), acetonitrile (Optima LC-MS), hexane, dichloromethane, chloroform, and acetone were purchased from Thermo-Fisher Scientific (FairLawn, NJ, USA). Ultrapure water was produced by a Mill-Q Reference system equipped with a LC-MS Pak filter (Millipore, Billerica, MA).

\subsection{Toxicological Study of PAR-Induced Liver Injury}

2.2.1. Zebrafish Larvae. Wild-type (AB strain) zebrafish after the fertilization of 96hpf was provided by the Beijing key lab for quality evaluation by Chinese Materia Medica of Beijing University of Chinese Medicine. The incubation procedures and culture conditions of zebrafish eggs were referred to the published protocol [16] and approved by the Animal Ethics Committee of Beijing University of Chinese Medicine.

2.2.2. Drug Exposure. Phytolacca acinosa Roxb (PAR) with batch number 200904 was provided by Anhui Guanghe Chinese Materia Medica Co., Ltd. (Anhui, China). PAR solutions with different solubility were prepared by double distilled water reflux extraction and stored at $-20^{\circ} \mathrm{C}$ for determination.
2.2.3. Lethality Curve. The zebrafish larvae from $96 \mathrm{hpf}$ after fertilization were randomly put into a 12 -well plate with 20 zebrafish in each well. $4 \mathrm{~mL}$ of the PAR liquid was added to each well, and 3 replicates were set in the experiment. The 12-well plate was placed in the standard culture environment and kept at $28.5 \pm 0.5^{\circ} \mathrm{C}$ for $14 / 10 \mathrm{~h}$ light-dark cycle. After PAR treatment for 24 hours, the death of zebrafish in each experimental group was observed and recorded under a DSQi2 fluorescence microscope (Nikon, Japan), and the average mortality of each group was calculated. SPSS20.0 was used to draw the best "mortality-concentration" effect curve, calculate the lethal concentration of the $10 \%$ zebrafish larvae $\left(\mathrm{LC}_{10}\right)$, and used the $\mathrm{LC}_{10}$ concentration in the rest of the experiments to provide a reference for the concentration setting of the target organ identification test.

2.2.4. Determination of ALT and AST. The zebrafish collected above were added to cold salt water $(w / v=1: 9)$, homogenized in an ice bath, centrifuged $\left(2500 \mathrm{rpm}, 4^{\circ} \mathrm{C}\right)$ for $10 \mathrm{~min}$, and the supernatant was taken according to the instructions provided in the detection kit (Nanjing Jiancheng Bioengineering Research Institute, Nanjing, China) for the determination of alanine aminotransferase (ALT) and aspartate aminotransferase (AST).

\subsubsection{Histopathological Evaluation of Hepatotoxicity.} Zebrafish larvae were fixed in $4 \%$ paraformaldehyde for 72 hours and rinsed with running water, the tissues were soaked in different concentrations of ethanol gradually dehydrated, transparent in xylene and immersed with paraffin, and embedded in paraffin specimens at $65^{\circ} \mathrm{C}$; the paraffin sections were cut by using a paraffin slicing machine $(4 \mu \mathrm{m} /$ piece). After drying, hematoxylin and eosin (H\&E) staining was used to observe the histopathological changes in zebrafish liver in the PAR-treated group and control group.

2.2.6. Detection of Necrosis and Apoptosis of Hepatocytes. 30 zebrafish larvae were randomly collected into an EP tube containing $1 \mathrm{~mL}$ dd $\mathrm{H}_{2} \mathrm{O}$ and $30 \mu \mathrm{L} 10 \mu \mathrm{g} / \mathrm{mL}$ AO staining solution was added to avoid light staining for $1 \mathrm{~h}$. The fluorescence in the zebrafish larvae were observed and photographed immediately by using a pose fluorescence microscope (Zeiss V16 Axio Zoom, $546 \mathrm{~nm}$ filter).

2.2.7. Statistical Analysis. Statistical analysis was carried out by SPSS 20.0, and the data were expressed as mean \pm standard deviation $(x \pm s)$. All data were accorded with normal distribution and the variance was homogeneous. The $\mathrm{LC}_{10}$ was calculated by one-way ANOVA and probability analysis. $P$ value $<0.05$ was considered to be statistically significant.

2.3. GC-MS Metabolomic Analysis. The sample preparation procedure was referred to the previously published method $[6,7]$. The metabolites in the supernatant of liver homogenate were analyzed by the gas chromatography-time-offlight-mass spectrometry (GC-TOF/MS) system, with 
Agilent 7890B gas chromatograph and a Gersted multipurpose sample MPS2 with dual heads (Muehlheim, Germany). A Rxi-5 MS capillary column $(30 \mathrm{~m} \times 250 \mu \mathrm{m}$, $0.25 \mu \mathrm{m}$, Bellefonte, USA) was used for separation. Helium was used as the carrier gas at a constant flow rate of $1.0 \mathrm{~mL} /$ min. Initially, the instrument was maintained at a temperature of $80^{\circ} \mathrm{C}$ and was retained for $2 \mathrm{~min}$. The temperature was increased to $300^{\circ} \mathrm{C}$ at a rate of $12^{\circ} \mathrm{C} / \mathrm{min}$ and was retained at $300^{\circ} \mathrm{C}$ for $4.5 \mathrm{~min}$. The temperature was increased from $300^{\circ} \mathrm{C}$ to $320^{\circ} \mathrm{C}$ at a rate of $40^{\circ} \mathrm{C} / \mathrm{min}$ and was retained at $320^{\circ} \mathrm{C}$ for $1 \mathrm{~min}$. The temperature of injection and the transfer interface were both set to $270^{\circ} \mathrm{C}$. The source temperature was $220^{\circ} \mathrm{C}$. The measurements were made by electron impact ionization $(70 \mathrm{eV})$ in the full-scan mode $(\mathrm{m} /$ $z$ 50-500). Instrument optimization was performed every 24 hours. 8 samples in each group were tested.

Nontargeted metabonomic analysis was carried out on the XploreMET platform (Metabo Profile, Shanghai, China). The biomarkers related to toxicity were screened and identified by principal component analysis (PCA) and orthogonal partial least square discriminant (OPLS-DA). The metabolic pathway enrichment and topological analysis of the differential metabolites were carried out by introducing MetaboAnalyst 4.0 to the study and analyzing the metabolic pathway of the biomarkers.

\subsection{Network Toxicological Study}

2.4.1. Potential Target Prediction of Hepatotoxicity. The chemical constituents of PAR were retrieved from CNKI and PUBMED and imported into the phytochemical database of Traditional Chinese Medicine Systems Pharmacology Database (TCMSP, https://tcmspw.com/index.php/))) [17] to screen the putative targets of PAR. The Universal Protein Resource (UniProt) (https://www.uniprot.org/)) was used to select target protein names that transformed into Gene Symbol and standardized correction with the species defined as human.

Using "hepatotoxicity, liver toxicity, and liver injury" as key words, disease targets were searched in Genecards (http://www.genecards.org/)), Toxicogenomics Database (CTD, http://ctdbase.org/), and OMIM (https://omim.org/) databases $[15,18]$. The data were cleaned and duplicated and were standardized by Uniprot database.

2.4.2. Protein-Protein Interaction (PPI). By using the online software mapping tool platform (http://www. bioinformatics.com.cn/login/), the component targets of PAR and liver toxicity related targets were mapped in Wayne diagram, and the intersection targets of PAR and hepatotoxicity were obtained, which were the key target of PAR and hepatotoxicity. The protein-protein interaction (PPI) network was constructed on the online platform of the STRING database (https://string- db.org/) [19]; the research species was defined as "Homo sapiens", and the minimum interaction score was set to "medium confidence" $(>0.4)$, while the other parameters were kept at the default setting.
2.4.3. GO and KEGG Enrichment Analysis. The PAR-hepatotoxicity common genes were introduced into DAVID [20] database (https://david.ncifcrf.gov/), entered the target gene name list and set species defined as "Homo Sapiens". GO analysis and KEGG [21] (Kyoto Encyclopedia of Genes and Genomes; https://www.kegg. Jp) pathway enrichment analysis was carried out, and enrichment results were visualized by the bioinformatics online tool to pick out the process and possible signal pathway of the PAR-induced liver injury.

\subsection{Molecular Biological Verification}

2.5.1. QT-PCR Analysis. 80 zebrafish larvae in each group were randomly selected and dissolved in Trizol reagent to extract total RNA. cDNA (complementary DNA) was synthesized by using a reverse transcription kit. TransStart Top Green and forward and reverse primers were used for real-time quantitative PCR (RT-PCR) analysis. The primer sequence is shown in Table 1 . The experiment was conducted 3 times with different batches of zebrafish.

2.5.2. Western Blot Test. Zebrafish whole fish $(n=80$ per concentration) was homogenized on ice, and equal amounts of protein quantified by the BCA method (Beijing TDY Biotechnology Co., Ltd., WB0028) were separated by $12 \%$ SDS-PAGE, transferred to a nitrocellulose filter membrane (NC) membrane, and then blocked with 3\%BSA-TBST. The primary antibody rabbit anti-caspase 3 (ab13847, abcam) was incubated at $4^{\circ} \mathrm{C}$ overnight, followed by TBST washing and incubation with secondary antibody goat anti-rabbit IgG $(H+L)$ HRP (Beijing TDY Biotech CO., Ltd. Beijing, S004) for $40 \mathrm{~min}$. After washing for $1 \mathrm{~min}$, it was reacted with ECL luminescent solution before exposure and was visualized by ChemiDoc XRS (Bio-Rad, Marnes-la-Coquette, France). The grayscale value of the exposure picture was analyzed by software ImageJ. The experiment was conducted 3 times with different batches of zebrafish.

\section{Results}

\subsection{Toxicologic Study of PAR-Induced Liver Injury}

3.1.1. Lethal Curve. At the experimental exposure end point, the mortality rate was calculated by counting the number of zebrafish surviving in different PAR-treated groups, and the dose-toxicity curve of PAR-induced hepatotoxicity on zebrafish was obtained. Logistic regression analysis was carried out by SPSS20.0 statistical software, drawing the dose-toxicity curve, and calculating the $\mathrm{LC}_{10}$ values. Results of the acute toxicity experiments showed that the mortality of zebrafish larvae increased in a concentration-dependent manner with the increasing PAR exposure concentration. As shown in Figure 1(a), the $\mathrm{LC}_{10}$ value of PAR calculated from the lethal curve was $1503.69 \mu \mathrm{g} / \mathrm{mL}$.

3.1.2. Liver Function Assessment. ALT and AST were amino acid transferases mainly distributed in hepatocytes, and they were commonly signal detection substances to evaluate liver 
TABLE 1: The sequences of the primers.

\begin{tabular}{lccc}
\hline Target gene & Accession ID & Forward primer sequence $\left(5^{\prime}-3^{\prime}\right)$ & Reverse primer sequence $\left(5^{\prime}-3^{\prime}\right)$ \\
\hline$\beta$-actin & AF057040.1 & GGCTGTGCTGTCCCTGTAT & GGGCGTAACCCTCGTAGAT \\
Caspase-3 & AB047003.1 & TCAGGCTTGTCGAGGAAC & CTGCCATACTTTGTCATCATTT \\
Caspase-8 & NM_131510.2 & AAGACCTGATTCTGCGACTG & TAGGCTGAGACACCTTTACG \\
Caspase-9 & NM_001007404.2 & TTCATCGCCCTCCTGTC & CTGGCATCCATCTTGTAGC \\
\hline
\end{tabular}

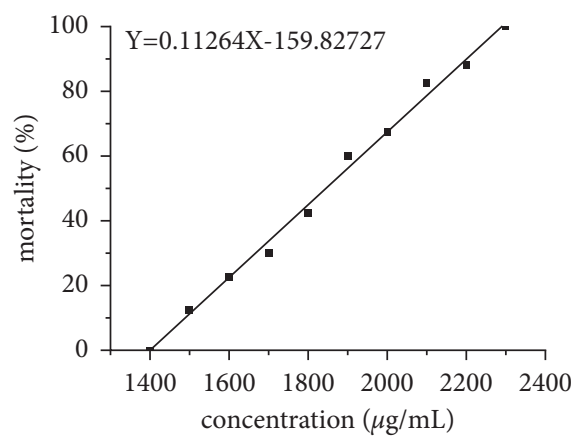

(a)
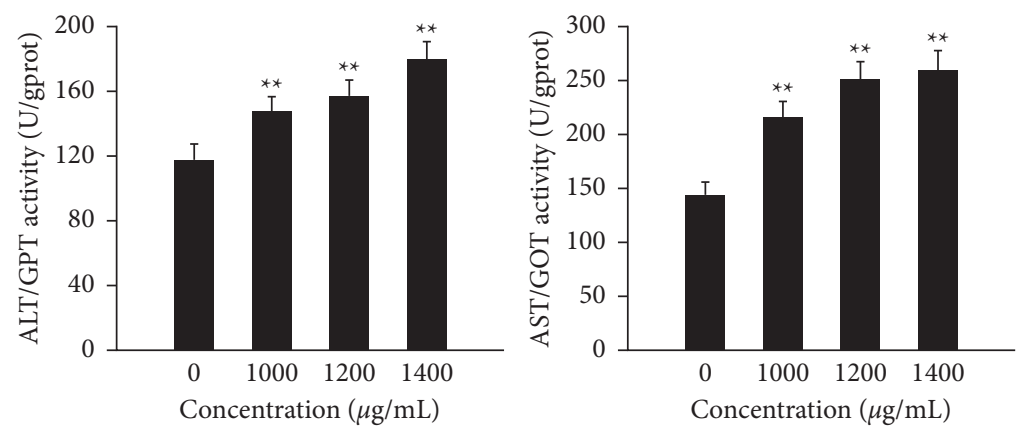

(b)

Figure 1: (a) Lethal curve from zebrafish induced by PAR from 96 hpf to 120 hpf. (b) The level of ALT and AST in zebrafish.

injury through exogenous substances. When hepatocytes were injured, the structure of hepatocytes was destroyed, and their transaminases would be released into blood circulation, causing both content to be increased in plasma. In this study, compared with the control group, ALT and AST values of zebrafish larvae exposed to PAR increased significantly as the concentration increased (Figure 1(b)). It is noteworthy that ALT activity was more sensitive to drug exposure, and the results were consistent with rodents and mammals, which indicated that PAR induced liver injury in zebrafish.

\subsubsection{Histopathological Evaluation of Hepatotoxicity in} Zebrafish. We studied the histopathological characteristics of zebrafish to evaluate the effect of PAR on hepatotoxicity. In the control group, hepatocytes were normal in the sections of zebrafish larvae with intact liver cells and were tightly arranged regularly (Figure 2(a)). In the PAR treatment group, the liver size and optical density of most zebrafish larvae changed significantly, the number of hepatocytes was reduced, and vacuolar degeneration and irregular arrangement appeared, which were consistent with the traditional endpoint time in published articles. PARtreated liver tissue cells exhibited obvious morphological changes and local necrosis, which showed PAR-induced liver injury (Figure 2(b)).

3.1.4. In Vivo Cell Death Assay Results. AO staining displayed hepatocyte necrosis and apoptosis of zebrafish larvae, while the control group and the PAR-treated group showed typical whole cell death images. The results revealed that the PAR-treated larvae had a significant inhibitory effect on apoptosis (Figure 3).
3.2. Metabolome Analysis. The unsupervised pattern recognition method, PCA analysis, can reflect the original state of the data and directly show the overall differences between different samples, so samples in the control group and PARtreated group were analyzed by PCA. It can be seen from Figure $4(\mathrm{a})$ that the samples of the two groups were significantly separated, indicating that there are some metabolic differences between the two groups.

With a significance level of 0.05 , a corresponding Corr. Value was used as a cutoff value to select the variables that were most correlated with the very first predictive components (Figure 4(a)). The diagnostic parameters of the PLS-DA model were summarized in Figure 4(a). Each point in the volcano chart represented a variable, and the importance of the variable in the classification was measured by the value of VIP (variable importance in the projection). The farther the variable was from the origin, the greater the VIP value.

The variables were screened according to the VIP value and $p$-value, and VIP $>1$ and $p<0.05$ were used as candidates for seeking differential metabolites. As shown in Table 2, 35 different metabolites were screened in this study. Compared with the control group, 9 substances were up-regulated (red in Table 2 ) and 26 substances were down-regulated (green in Table 2) in the samples of the administration group, which might suggest that these 35 endogenous metabolites were related to the liver function damage of zebrafish induced by PAR. Based on the KEGG enrichment analysis, arginine biosynthesis, arginine and proline metabolism (Figure 4(b)), taurine and hypotaurine metabolism, and cysteine and methionine metabolism were selected as potential pathways by using the MetaboAnalyst 3.0 online system (MetPA, https://www. metaboanalyst.ca/). 


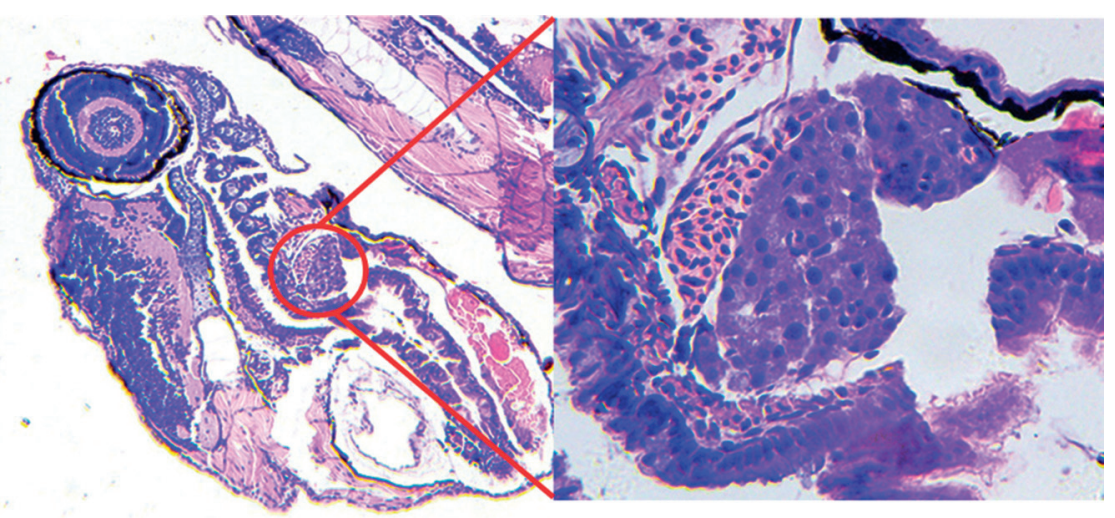

(a)

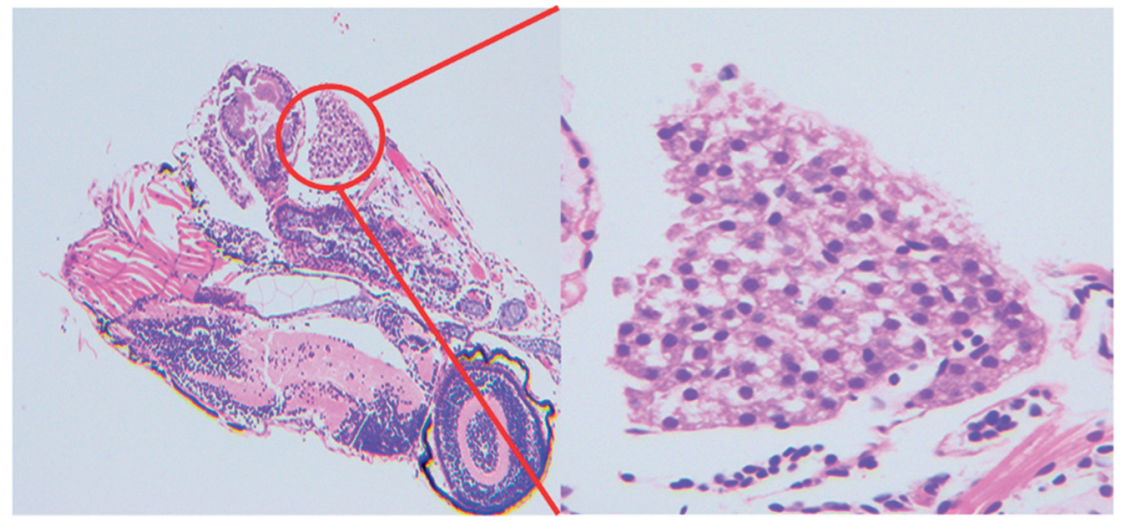

(b)

Figure 2: Histopathology of zebrafish liver in both control group and PAR-treated group. (a) Control (100×, 400×). (b) PAR (100×, 4 00×).

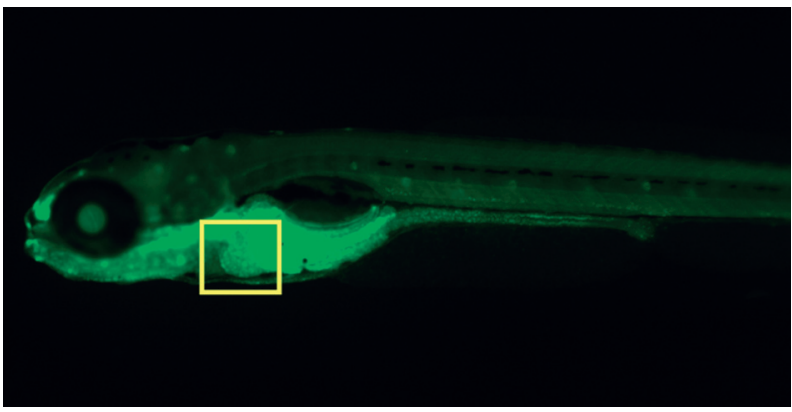

(a)

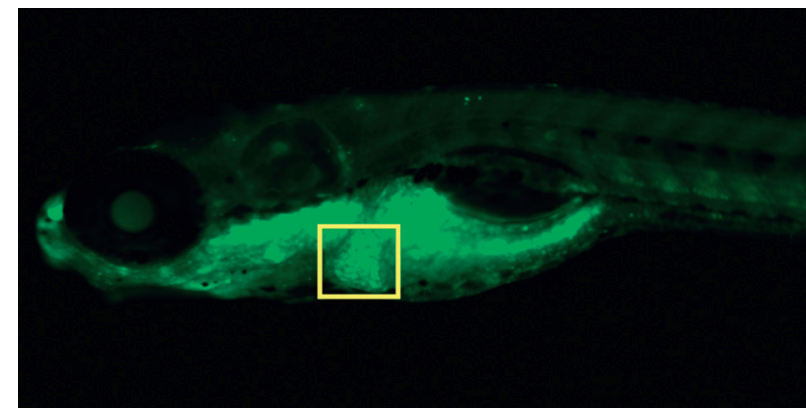

(b)

FIGURE 3: In vivo cell death and apoptosis results. (a) Control group. (b) PAR group.

\subsection{Network Toxicological Study}

3.3.1. Potential Target Prediction of Hepatotoxicity. The chemical constituents of PAR retrieved from CNKI and PUBMED databases were all imported into TCMSP database for retrieval. 11 compounds with $\mathrm{OB} \geq 30 \%$ and $\mathrm{DL} \geq 0.18$ were screened out according to oral bioavailability and druglike analysis, and a total of 92 targets were obtained in the TCMSP database. The PAR component-target network consists of 92 target nodes and 11 active component nodes (Figure 5).
All the hepatotoxic related targets were searched through Genecards, TCD, and OMIM, the duplicates were deleted and 8476 target proteins were integrated, and all the predicted target proteins were converted into corresponding gene names through Uniprot database.

3.3.2. Protein-Protein Interaction (PPI). To further explore the mechanism of hepatotoxicity induced by PAR, we found that 79 targets were overlapped in both PAR active components and hepatotoxic targets (Figure 6(a)). These 79 

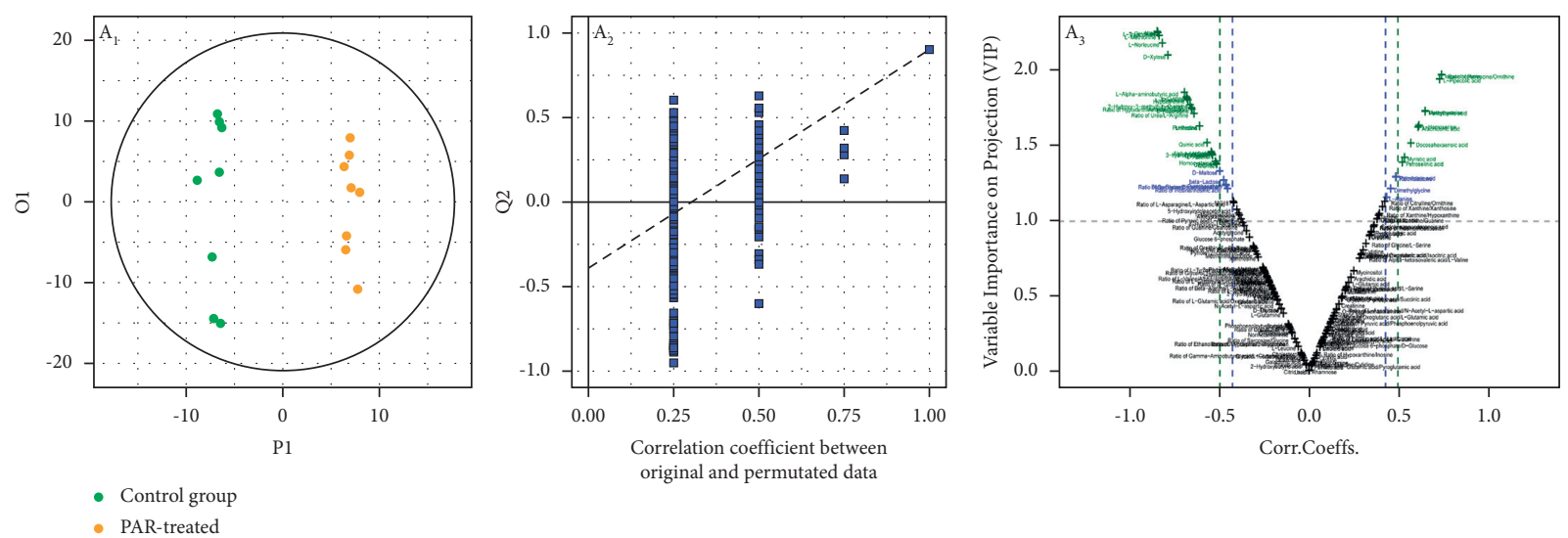

- PAR-treated

(a)

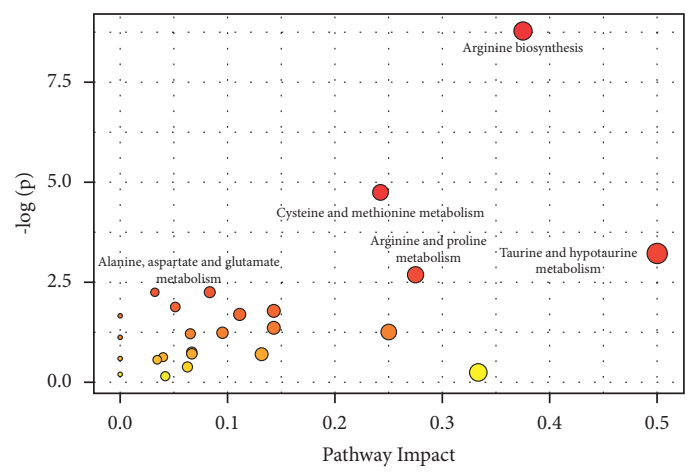

(b)

FIgURE 4: (a) Visualization of the overall metabolite profile difference between the PAR-treated group and the control group including the OPLS-DA predictive/discriminate score plot $\left(a_{1}\right), 1000$ permutation tests $\left(a_{2}\right)$, and V-plot $\left(a_{3}\right)$; $(b)$ differential metabolic pathways between the PAR-treated group and the control group.

targets were imported into STRING database to analyze the relationship between proteins, and the PPI network was obtained (Figure 6(c)). In the network, nodes represent targets, edges represent the interaction between targets, and different colors represent different interactions. The PPI network was analyzed by Cytoscape3.7.2, the degree value median was 9.5, and the average topology coefficient was 0.350 . According to the degree values, 16 core targets with degree $>19$ were screened, namely, CASP3, TNF, IL6, MMP9, NOS3, HSP90AA1, CASP8, PTGS2, AR, SP1, PTEN, CDK2, ICAM1, CDK4, CASP9, and HMOX1; for specific information, see Figure 6(b). These may be potential targets for PAR-induced hepatotoxicity.

3.3.3. GO and KEGG Enrichment Analysis. The DAVID database was used to analyze the functional enrichment of 78 PPI targets. In the GO functional enrichment analysis, the first 10 items of the biological process, cellular composition, and molecular function were selected (Table 3). The biological process involved negative regulation of calcium ion transport, positive regulation of vasoconstriction, and negative regulation of apoptotic process; the cellular component involves caveola, membrane raft, asymmetric synapse etc.; molecular function mainly involves protein homodimerization activity, nitric-oxide synthase activity, norepinephrine binding, etc.
In the KEGG pathway enrichment analysis, the first 20 items were selected to draw a bar chart (Figure 6(d)). The main pathways related to hepatotoxicity are metabolic pathway, namely, arginine and proline metabolism; PI3K-Akt signaling pathway in environmental information procession; p53 signaling pathway and TNF signaling pathway in cellular processes; and apoptosis pathway. Based on the above results of cyber toxicological studies on these core targets, biological processes, and signal pathways on PAR-induced hepatotoxicity, it was found that the screened out active components of PAR with core targets exerted hepatotoxicity effects that were related to a variety of biological processes, whose mechanisms may be closely related to the regulation of physiological processes such as apoptosis, cell proliferation, and oxidative stress.

\subsection{Molecular Biology Verification}

3.4.1. QT-PCR Analysis of Key Target Genes. The P53 signal pathway and apoptosis signal pathway are located on the same signal axis. Because the apoptosis signal pathway was a key factor in tissue injury, and the caspase family played an important role in the regulation of apoptosis, we used different concentrations of PAR to induce zebrafish to detect the transcriptional levels of caspase-3, caspase-8, and 
TABLE 2: 35 metabolites contributing to the separation between the control and treated groups exposed to PAR $(p<0.05)$.

\begin{tabular}{|c|c|c|c|c|c|c|c|c|c|}
\hline Class & Metabolites & Formula & HMDBID & KEGGID & VIP & $P$-value & FC & Corr.Coeffs. & Effect \\
\hline Amino acid & Urea & $\mathrm{CH}_{4} \mathrm{~N}_{2} \mathrm{O}$ & HMDB00294 & C00086 & 2.2 & $6.49 E-04$ & 0.6 & -0.84 & $\downarrow$ \\
\hline Amino acid & Ornithine & $\mathrm{C}_{5} \mathrm{H}_{12} \mathrm{~N}_{2} \mathrm{O}_{2}$ & HMDB00214 & C00077 & 2.2 & $7.21 E-05$ & 0.7 & -0.84 & $\downarrow$ \\
\hline Amino acid & L-Tryptophan & $\mathrm{C}_{11} \mathrm{H}_{12} \mathrm{~N}_{2} \mathrm{O}_{2}$ & HMDB00929 & $\mathrm{C} 00078$ & 2.2 & $3.08 E-04$ & 0.8 & -0.84 & $\downarrow$ \\
\hline Amino acid & L-Methionine & $\mathrm{C}_{5} \mathrm{H}_{11} \mathrm{NO}_{2} \mathrm{~S}$ & HMDB00696 & C00073 & 2.2 & $1.56 E-04$ & 0.8 & -0.83 & $\downarrow$ \\
\hline Amino acid & L-norleucine & $\mathrm{C}_{6} \mathrm{H}_{13} \mathrm{NO}_{2}$ & HMDB01645 & C01933 & 2.2 & $2.95 E-04$ & 0.7 & -0.82 & $\downarrow$ \\
\hline Carbohydrates & D-Xylose & $\mathrm{C}_{5} \mathrm{H}_{10} \mathrm{O}_{5}$ & HMDB00098 & C00181 & 2.1 & $7.02 E-04$ & 0.6 & -0.79 & $\downarrow$ \\
\hline Nucleotide & Isoxanthopterin & $\mathrm{C}_{6} \mathrm{H}_{5} \mathrm{~N}_{5} \mathrm{O}_{2}$ & HMDB00704 & C03975 & 2.0 & $1.25 E-03$ & 1.7 & 0.74 & $\uparrow$ \\
\hline Amino acid & $\begin{array}{l}\text { Ratio of putrescine/ } \\
\text { ornithine }\end{array}$ & $\begin{array}{c}\mathrm{C}_{4} \mathrm{H}_{12} \mathrm{~N}_{2} / \\
\mathrm{C}_{5} \mathrm{H}_{12} \mathrm{~N}_{2} \mathrm{O}_{2}\end{array}$ & $\begin{array}{l}\text { HMDB01414/ } \\
\text { HMDB00214 }\end{array}$ & $\begin{array}{l}\text { C00134/ } \\
\text { C00077 }\end{array}$ & 2.0 & $1.71 E-03$ & 1.1 & 0.74 & $\uparrow$ \\
\hline Organic acids & L-Pipecolic acid & $\mathrm{C}_{6} \mathrm{H}_{11} \mathrm{NO}_{2}$ & HMDB00716 & $\mathrm{C} 00408$ & 1.9 & $2.52 E-03$ & 2.5 & 0.73 & $\uparrow$ \\
\hline Amino acid & $\begin{array}{l}\text { L-Alpha-aminobutyric } \\
\text { acid }\end{array}$ & $\mathrm{C}_{4} \mathrm{H}_{9} \mathrm{NO}_{2}$ & HMDB00452 & C02356 & 1.8 & $4.70 E-03$ & 0.8 & -0.69 & $\downarrow$ \\
\hline Amino acid & L-Cystine & $\mathrm{C}_{6} \mathrm{H}_{12} \mathrm{~N}_{2} \mathrm{O}_{4} \mathrm{~S}_{2}$ & HMDB00192 & C00491 & 1.8 & $3.92 E-03$ & 0.7 & -0.68 & $\downarrow$ \\
\hline Amino acid & L-Threonine & $\mathrm{C}_{4} \mathrm{H}_{9} \mathrm{NO}_{3}$ & HMDB00167 & C00188 & 1.8 & $8.37 E-03$ & 0.8 & -0.68 & $\downarrow$ \\
\hline Nucleotide & Hypoxanthine & $\mathrm{C}_{5} \mathrm{H}_{4} \mathrm{~N}_{4} \mathrm{O}$ & HMDB00157 & C00262 & 1.8 & $5.34 E-03$ & 0.9 & -0.67 & $\downarrow$ \\
\hline Amino acid & L-Cysteine & $\mathrm{C}_{3} \mathrm{H}_{7} \mathrm{NO}_{2} \mathrm{~S}$ & HMDB00574 & C00097 & 1.8 & $1.10 E-02$ & 0.8 & -0.66 & $\downarrow$ \\
\hline Organic acids & $\begin{array}{l}\text { 2-Hydroxy-3- } \\
\text { methylbutyric acid }\end{array}$ & $\mathrm{C}_{5} \mathrm{H}_{10} \mathrm{O}_{3}$ & HMDB00407 & NA & 1.8 & $7.05 E-03$ & 0.8 & -0.66 & $\downarrow$ \\
\hline Amino acid & 2-Aminoadipate & $\mathrm{C}_{6} \mathrm{H}_{11} \mathrm{NO}_{4}$ & HMDB00510 & C00956 & 1.7 & $8.61 E-03$ & 0.8 & -0.65 & $\downarrow$ \\
\hline Nucleotide & $\begin{array}{c}\text { Ratio of hypoxanthine/ } \\
\text { inosinic acid }\end{array}$ & $\begin{array}{c}\mathrm{C}_{5} \mathrm{H}_{4} \mathrm{~N}_{4} \mathrm{O} / \\
\mathrm{C}_{10} \mathrm{H}_{13} \mathrm{~N}_{4} \mathrm{O}_{8} \mathrm{P}\end{array}$ & $\begin{array}{l}\text { HMDB00157/ } \\
\text { HMDB00175 }\end{array}$ & $\begin{array}{l}\mathrm{C} 00262 / \\
\mathrm{C} 00130\end{array}$ & 1.7 & $5.96 E-03$ & 0.7 & -0.65 & $\downarrow$ \\
\hline Amino acid & L-Asparagine & $\mathrm{C}_{4} \mathrm{H}_{8} \mathrm{~N}_{2} \mathrm{O}_{3}$ & HMDB00168 & C00152 & 1.7 & $9.63 E-03$ & 0.6 & -0.65 & $\downarrow$ \\
\hline Amino acid & Methylcysteine & $\mathrm{C}_{4} \mathrm{H}_{9} \mathrm{NO}_{2} \mathrm{~S}$ & HMDB02108 & NA & 1.7 & $1.38 E-02$ & 2 & 0.65 & $\uparrow$ \\
\hline Vitamin & Pantothenic acid & $\mathrm{C}_{9} \mathrm{H}_{17} \mathrm{NO}_{5}$ & HMDB00210 & C00864 & 1.7 & $6.53 E-03$ & 1.4 & 0.65 & $\uparrow$ \\
\hline Amino acid & Ratio of urea/L-arginine & $\begin{array}{c}\mathrm{CH}_{4} \mathrm{~N}_{2} \mathrm{O} / \\
\mathrm{C}_{6} \mathrm{H}_{14} \mathrm{~N}_{4} \mathrm{O}_{2}\end{array}$ & $\begin{array}{l}\text { HMDB00294/ } \\
\text { HMDB00517 }\end{array}$ & $\begin{array}{l}\mathrm{C} 00086 / \\
\mathrm{C} 00062\end{array}$ & 1.7 & $9.49 E-03$ & 0.7 & -0 & $\downarrow$ \\
\hline Alkylamines & Putrescine & $\mathrm{C}_{4} \mathrm{H}_{12} \mathrm{~N}_{2}$ & HMDB01414 & $\mathrm{C} 00134$ & 1.6 & 0.0159 & 0.8 & -0.61 & $\downarrow$ \\
\hline Amino acid & L-Homoserine & $\mathrm{C}_{4} \mathrm{H}_{9} \mathrm{NO}_{3}$ & HMDB00719 & $\mathrm{C} 00263$ & 1.6 & 0.0171 & 2.3 & 0.61 & $\downarrow$ \\
\hline Amino acid & L-Proline & $\mathrm{C}_{5} \mathrm{H}_{9} \mathrm{NO}_{2}$ & HMDB00162 & $\mathrm{C} 00148$ & 1.6 & 0.0237 & 0.9 & -0.61 & $\downarrow$ \\
\hline Fatty acids & Arachidonic acid & $\mathrm{C}_{20} \mathrm{H}_{32} \mathrm{O}_{2}$ & HMDB01043 & C00219 & 1.6 & 0.0113 & 1.4 & 0.61 & $\uparrow$ \\
\hline Fatty acids & Docosahexaenoic acid & $\mathrm{C}_{22} \mathrm{H}_{32} \mathrm{O}_{2}$ & HMDB02183 & C06429 & 1.5 & 0.0248 & 1.3 & 0.57 & $\uparrow$ \\
\hline Organic acids & Quinic acid & $\mathrm{C}_{7} \mathrm{H}_{12} \mathrm{O}_{6}$ & HMDB03072 & C06746 & 1.5 & 0.0238 & 0.4 & -0.57 & $\downarrow$ \\
\hline Carbohydrates & Alpha-lactose & $\mathrm{C}_{12} \mathrm{H}_{22} \mathrm{O}_{11}$ & HMDB00186 & $\mathrm{C} 00243$ & 1.4 & 0.0432 & 0.7 & -0.54 & $\downarrow$ \\
\hline Lipids & MG160 & $\mathrm{C}_{19} \mathrm{H}_{38} \mathrm{O}_{4}$ & HMDB11564 & NA & 1.4 & 0.0353 & 0.9 & -0.54 & $\downarrow$ \\
\hline NA & 3-Hydroxypyridine & $\mathrm{C}_{5} \mathrm{H}_{5} \mathrm{NO}$ & NA & NA & 1.4 & 0.0359 & 0.9 & -0.54 & $\downarrow$ \\
\hline Carbohydrates & L-Arabitol & $\mathrm{C}_{5} \mathrm{H}_{12} \mathrm{O}_{5}$ & HMDB01851 & C00532 & 1.4 & 0.0344 & 0.9 & -0.54 & $\downarrow$ \\
\hline Fatty acids & Myristic acid & $\mathrm{C}_{14} \mathrm{H}_{28} \mathrm{O}_{2}$ & HMDB00806 & $\mathrm{C} 06424$ & 1.4 & 0.0405 & 1.2 & 0.53 & $\uparrow$ \\
\hline Amino acid & Homocysteine & $\mathrm{C}_{4} \mathrm{H}_{9} \mathrm{NO}_{2} \mathrm{~S}$ & HMDB00742 & C00155 & 1.4 & 0.0367 & 0.8 & -0.52 & $\downarrow$ \\
\hline Organic acids & Petroselinic acid & $\mathrm{C}_{18} \mathrm{H}_{34} \mathrm{O}_{2}$ & HMDB02080 & C08363 & 1.4 & 0.0334 & 1.4 & 0.52 & $\uparrow$ \\
\hline Lipids & MG182 & $\mathrm{C}_{21} \mathrm{H}_{38} \mathrm{O}_{4}$ & HMDB11568 & NA & 1.4 & 0.0477 & 0.7 & -0.51 & $\downarrow$ \\
\hline
\end{tabular}

caspase-9. As shown in Figure 7(a), compared with the normal control group, the PAR of $1000-1400 \mu \mathrm{g} / \mathrm{mL}$ had significant effects on caspase 3 , caspase- 8 , and caspase- 9 genes of zebrafish. The expression levels of caspase- 3 and caspase- 8 were significantly increased at $1000-1400 \mu \mathrm{g} / \mathrm{mL}$, while the expression of caspase- 9 was significantly increased when the concentration of PAR was $1200-1400 \mu \mathrm{g} / \mathrm{mL}$. The mentioned results revealed that hepatocyte apoptosis and hepatotoxicity occurred in zebrafish hepatocytes within $4 \mathrm{dpf}$ after the administration of PAR.

3.4.2. Western Blot Analysis of Caps3 Protein. The molecular mechanism of apoptosis according to whether it depended on Caspase or not can be divided into two types, Caspasedependent and Caspase-independent. The former is the most classical apoptosis pathway, and the vast majority of cells induce apoptosis by activating Caspase. Among the
Caspase family proteins, Caps3 was the downstream of the apoptosis regulation pathway, which was the key executive molecule in the apoptosis signal transduction pathway, so it is also called the death protease. To further detect the expression level of protein Caps3, we used western blot to detect the protein expression of Caps3 in zebrafish. As shown in Figure 7(b), the expression of Caps3 in the liver of zebrafish treated with PAR was significantly up-regulated, indicating that PAR induced hepatotoxicity in zebrafish in a Caspase-dependent manner by activating Caps3.

\section{Discussion}

Over the course of the past decades, there has been a worldwide effort aimed at developing drugs from natural products to treat various health conditions and tend to make the TCM as potential therapeutic options of worldwide importance. The reasons for this phenomenon may be 


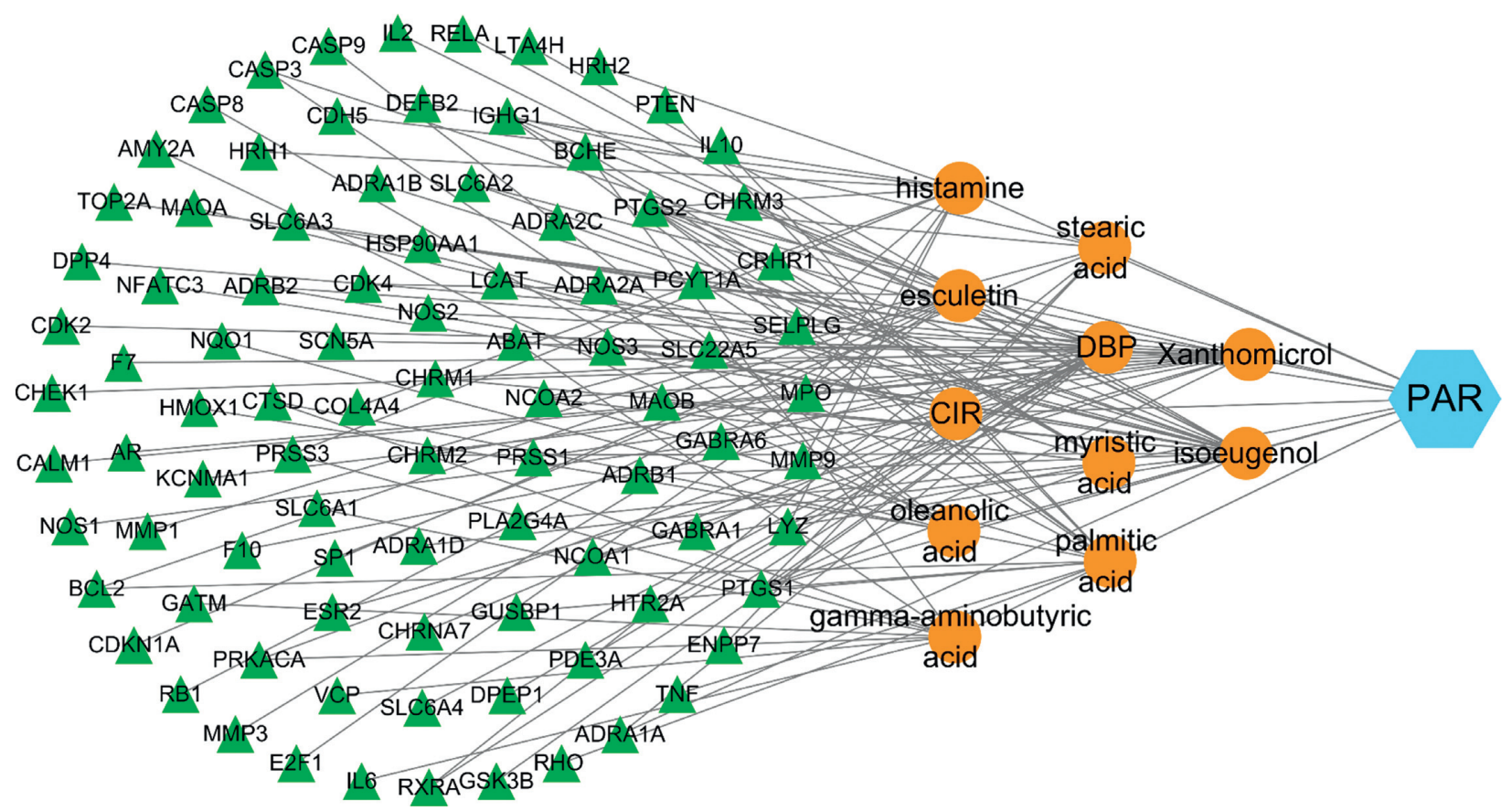

FIGURE 5: Network construction of the "PAR-component-target (note: the orange nodes represent the PAR component and green nodes represent the targets of the PAR component).

related to a belief that herbal TCMs labeled as "natural" are always safe and of benefit to public health, improving physical fitness, extending lifespan, and treating various illnesses. Recently, the abundance of the reported liver injury cases induced by the TCM has attracted wide public and regulatory attention, especially related to hepatotoxicity, which can evolve into acute liver failure in most serious cases $[22,23]$. It is known that hepatotoxicity results from a sequence of induced mechanisms and is involved with different liver toxic phenotypes, such as cholestasis [24], steatosis [25], and necrosis because of different external factors, which brings great difficulties in the definitive diagnosis of most TCM-induced hepatotoxicity in the clinic and drug development.

In this study, we used zebrafish as an animal model [26-28] to observe the liver morphology of the zebrafish induced by PAR, the changes of biochemical indexes of liver function enzymes, and the effect of AO staining on hepatotoxicity. Combined with nontargeted metabolites by using GC-MS, network toxicology and molecular biology methods were used to find out differential metabolites and metabolic pathways to elucidate the potential mechanism of zebrafish hepatotoxicity induced by PAR.

To evaluate the hepatotoxicity of PAR, we performed tissue sections and AO staining on whole zebrafish to determine whether PAR could destroy the liver. At the same time, acute toxicity experiments were carried out to observe the lethality of zebrafish treated with different PAR concentrations. Compared with the control group, the PARtreated group exhibited green fluorescence, which indicated that hepatocytes were obviously apoptotic after AO staining. We found some changes in hepatocytes including the decrease in number, vacuolar degeneration, irregular arrangement, destruction of cell structure, and even degeneration. As ALT and AST are the common indicators for the clinical evaluation of hepatotoxicity, ALT and AST levels in the PAR-exposure group were significantly increased than those in the control group $(p<0.05)$. Therefore, ALT and AST accumulated in the zebrafish liver by the induction of PAR, resulting in hepatocyte damage and necrosis.

Nontargeted metabolomics were applied for seeking different metabolites and probable metabolic pathways. In this study, we found that the metabolic pathways arginine biosynthesis, arginine and proline metabolism, taurine and hypotaurine metabolism, cysteine and methionine metabolism were perturbed by PAR. Methionine protected liver mainly related to the following two aspects: (1) promoting phospholipid methylation of hepatocyte membrane, increasing membrane fluidity, and reducing cholestasis in hepatocytes and (2) strengthening the trans-sulfuration function, so as to accelerate the synthesis of cysteine, glutathione, and taurine in hepatocytes, and improve the antioxidant capacity of organism [29, 30]. Methionine can induce endogenous antioxidant response by activating the Nrf2-ARE pathway, while in turn improving the ROS-derived oxidative stress. In this study, both cysteine and methionine on PAR-treated zebrafish liver were decreased significantly, which might inhibit the sulfur transfer pathway of methionine, causing abnormal metabolism of cysteine and methionine, and leading to redox imbalance; therefore, hepatocytes were more vulnerable to attack and damage by free radicals.

The metabolism of proline was down-regulated, indicating that the arginine and proline metabolism was disturbed. Proline is a nonessential amino acid, mainly synthesized by glutamate, which plays important roles in 


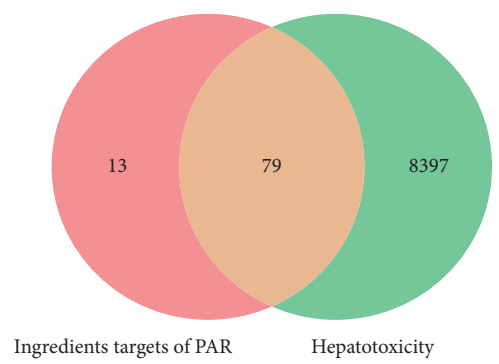

(a)

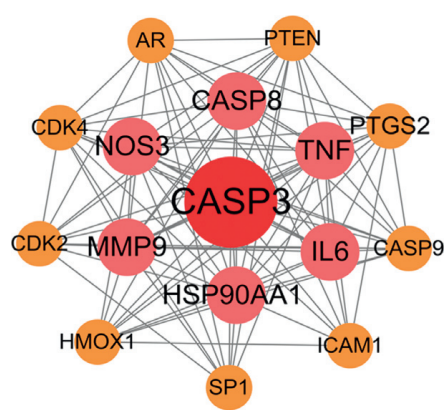

(b)

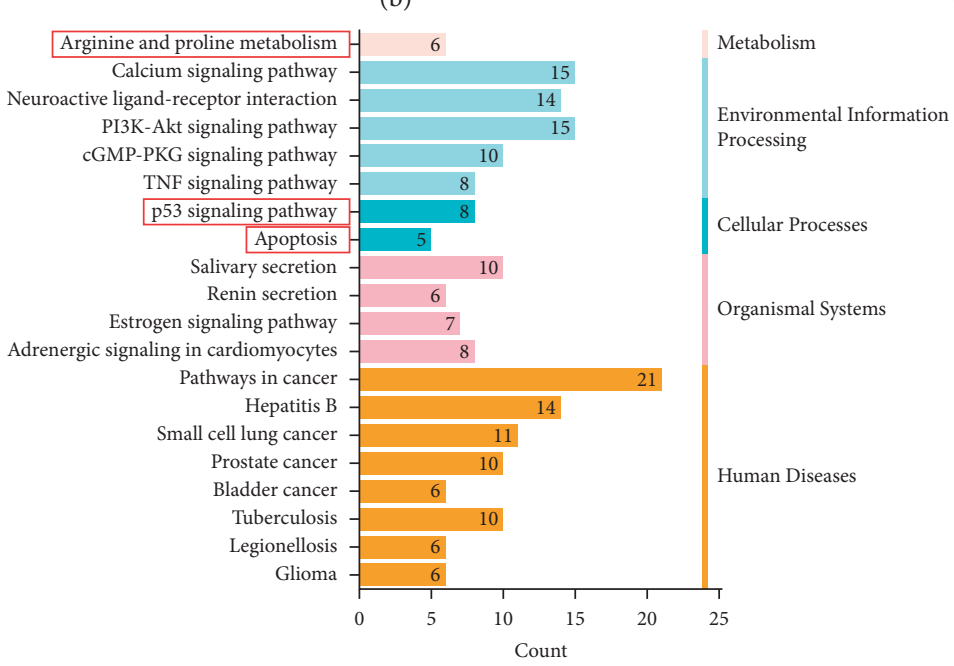

(d)

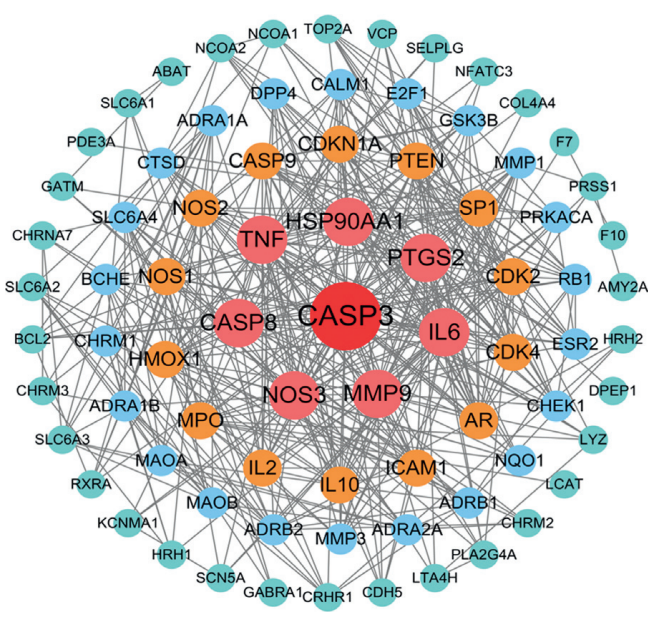

(c) 
TABLe 3: GO analysis of potential targets.

\begin{tabular}{|c|c|c|c|c|}
\hline GO ID & Terms & Subgroup & Gene counts & $\mathrm{P}$-value \\
\hline GO:0042493 & Response to drug & Biological process & 18 & $3.58 \times 10^{-14}$ \\
\hline GO:0032355 & Response to estradiol & Biological process & 10 & $3.61 \times 10^{-10}$ \\
\hline GO:0001666 & Response to hypoxia & Biological process & 10 & $1.00 \times 10^{-7}$ \\
\hline GO:0051926 & Negative regulation of calcium ion transport & Biological process & 5 & $2.98 \times 10^{-7}$ \\
\hline GO:0045907 & Positive regulation of vasoconstriction & Biological process & 6 & $3.47 \times 10^{-7}$ \\
\hline GO:0051384 & Response to glucocorticoid & Biological process & 7 & $5.49 \times 10^{-19}$ \\
\hline GO:0045471 & Response to ethanol & Biological process & 8 & $5.61 \times 10^{-18}$ \\
\hline GO:0035094 & Response to nicotine & Biological process & 6 & $7.37 \times 10^{-7}$ \\
\hline GO:0007568 & Aging & Biological process & 9 & $9.59 \times 10^{-7}$ \\
\hline GO:0043066 & Negative regulation of apoptotic process & Biological process & 13 & $1.16 \times 10^{-6}$ \\
\hline GO:0045121 & Membrane raft & Cellular component & 10 & $2.35 \times 10^{-7}$ \\
\hline GO:0043005 & Neuron projection & Cellular component & 10 & $1.18 \times 10^{-6}$ \\
\hline GO:0005886 & Plasma membrane & Cellular component & 38 & $5.79 \times 10^{-6}$ \\
\hline GO:0005887 & Integral component of plasma membrane & Cellular component & 20 & $8.52 \times 10^{-6}$ \\
\hline GO:0005901 & Caveola & Cellular component & 6 & $3.60 \times 10^{-5}$ \\
\hline GO:0005576 & Extracellular region & Cellular component & 20 & $3.60 \times 10^{-5}$ \\
\hline GO:0016324 & Apical plasma membrane & Cellular component & 8 & $2.47 \times 10^{-4}$ \\
\hline GO:0032279 & Asymmetric synapse & Cellular component & 3 & $4.98 \times 10^{-4}$ \\
\hline GO:0005615 & Extracellular space & Cellular component & 16 & $5.18 \times 10^{-4}$ \\
\hline GO:0000307 & Cyclin-dependent protein kinase holoenzyme complex & Cellular component & 3 & 0.00183 \\
\hline GO:0019899 & Enzyme binding & Molecular function & 10 & $2.24 \times 10^{-5}$ \\
\hline GO:0008144 & Drug binding & Molecular function & 6 & $2.64 \times 10^{-5}$ \\
\hline GO:0042803 & Protein homodimerization activity & Molecular function & 14 & $2.73 \times 10^{-5}$ \\
\hline GO:0004517 & Nitric-oxide synthase activity & Molecular function & 3 & $6.30 \times 10^{-5}$ \\
\hline GO:0051380 & Norepinephrine binding & Molecular function & 3 & $1.26 \times 10^{-4}$ \\
\hline GO:0034617 & Tetrahydrobiopterin binding & Molecular function & 3 & $1.26 \times 10^{-4}$ \\
\hline GO:0004252 & Serine-type endopeptidase activity & Molecular function & 8 & $1.75 \times 10^{-4}$ \\
\hline GO:0051379 & Epinephrine binding & Molecular function & 3 & $3.12 \times 10^{-4}$ \\
\hline GO:0031625 & Ubiquitin protein ligase binding & Molecular function & 8 & $3.60 \times 10^{-4}$ \\
\hline GO:0020037 & Heme binding & Molecular function & 6 & $4.30 \times 10^{-4}$ \\
\hline
\end{tabular}
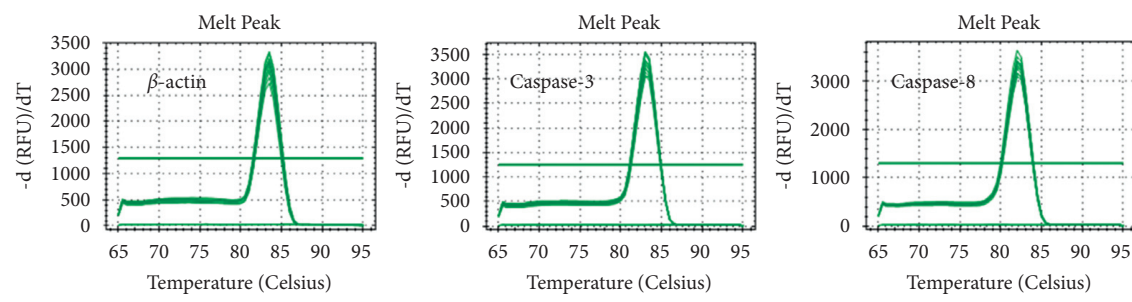

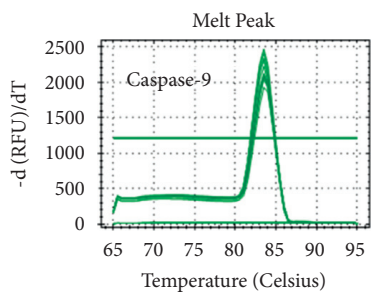

(a)
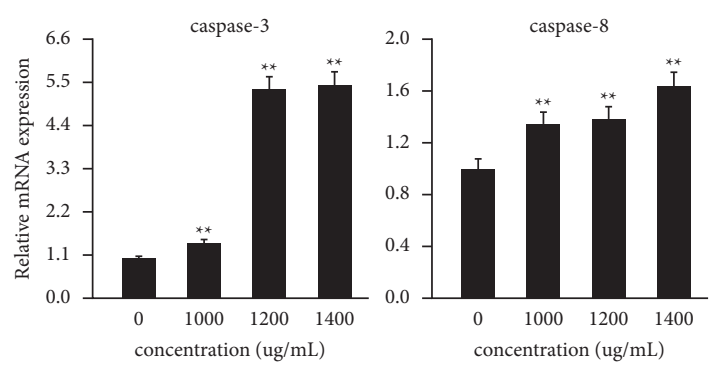

(b)

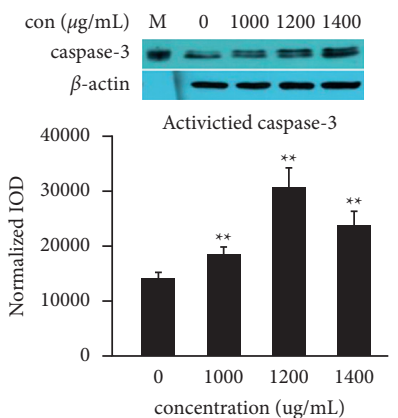

(c)

Figure 7: (a, b) Gene expression of caspase-3, caspase-8, and caspase-9 was examined by QT-PCR in groups treated with PAR. (c) Protein expression of activated Caps 3 was examined by western blot in the zebrafish larvae group treated with PAR, $n=80 .{ }^{*}$ indicates $p<0.05$ versus the control group, ${ }^{* *}$ indicates $p<0.01$ versus the control group, by one-way ANOVA. 


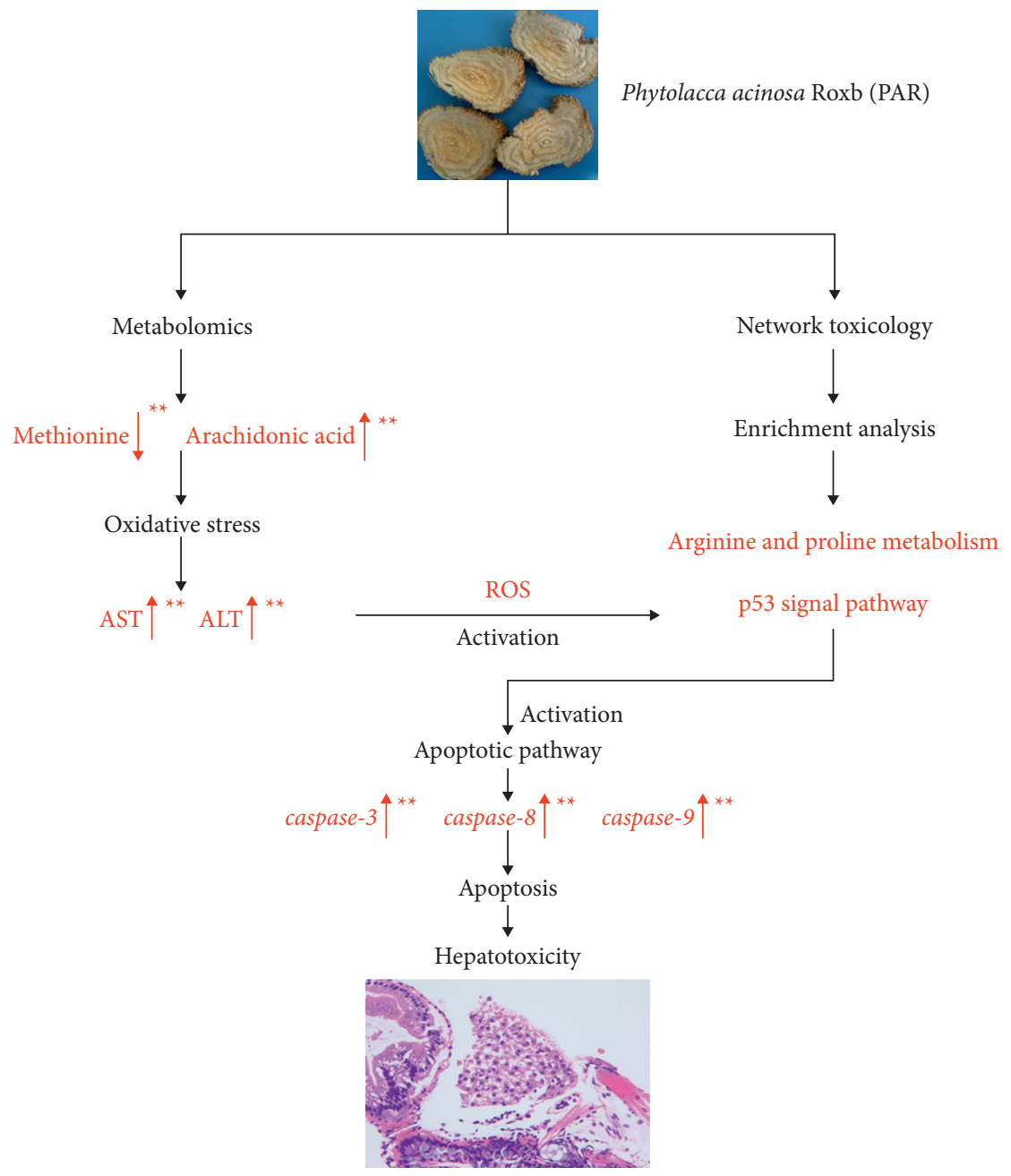

Figure 8: Molecular mechanism of PAR-induced hepatotoxicity.

occurred after zebrafish was treated with PAR, and caspase3, caspase-8, and caspase-9 were significantly up-regulated, which also verified the prediction via network toxicology.

Our results demonstrate a strong correlation between PAR and liver injury. Compared with the control group, the liver tissue of zebrafish in the PAR exposure group had pathological changes, and the liver function test suggested that PAR could cause hepatotoxicity. The metabolic disorder of amino acid was found to up-regulate arachidonic acid through metabonomics, and network toxicology was used to screen the key signal pathways (p53 signal pathway and arginine and proline metabolism). High levels of arachidonic acid and descendant methionine can trigger oxidative stress and excessive ROS, which may activate the $\mathrm{p} 53$ pathway and cause apoptosis. The overexpression of caspase-3, caspase-8, and caspase-9 can induce hepatocyte apoptosis and eventually resulted in liver injury (Figure 8).

\section{Conclusion}

The molecular mechanism of hepatotoxicity induced by PAR was analyzed by nontargeted metabolomics, molecular biology, and network toxicology. First, a comprehensive analysis of liver alterations, including liver morphology and function of zebrafish liver was observed. Subsequently, the changes in endogenous metabolites, especially amino acids analysis and network toxicology, were predicted. Finally, the genes and proteins related to liver injury were verified. Besides, this study provides the basis for the use of zebrafish in the prediction of drug hepatotoxicity and reference for future studies on the hepatotoxicity mechanism in other models and supply data for its safety on clinical use.

\section{Abbreviations}

TCM: Traditional Chinese Medicine

PAR: Phytolacca acinosa Roxb

HK-2: Hexokinase 2

ALT: $\quad$ Alanine transaminase

AST: $\quad$ Aspartate transaminase

PBS: $\quad$ Phosphate buffer saline

H\&E: Hematoxylin and eosin

AO: Acridine orange

GC-MS: Gas chromatography-mass spectrometer

PCA: Principal component analysis

PLS-DA: Partial least squares discriminant analysis 
KEGG: Kyoto Encyclopedia of Genes and Genomes

DAVID: Database for Annotation Visualization and Integrated Discovery

TCMSP: Traditional Chinese Medicine Systems Pharmacology

CNKI: Chinese National Knowledge Infrastructure

UniProt: Universal Protein Resource

GO: $\quad$ Gene Ontology

PPI: Protein-Protein Interaction

CC: $\quad$ Cellular Component

BP: Biological Process

MF: Molecular Function

FC: $\quad$ Fold changes

VIP: Variable importance in projection

XOD: Xanthine oxidase

COX: Cyclooxygenase

LOX: Lipoxygenase.

\section{Data Availability}

The data used to support the findings of this study are included within the article.

\section{Conflicts of Interest}

The authors declare that the research was conducted in the absence of any commercial and financial relationships that could be constructed as a potential conflict of interest.

\section{Authors' Contributions}

DC and CJZ conceived the project. JL and RCL provided supervision. MLC, HYW, and YYJ performed the research. ZQL, QQF, and HJN analyzed the data. DC, JL, and CJZ wrote the article. Dan Cao and Chongjun Zhao contributed equally to this work.

\section{Acknowledgments}

This study was supported in part by the National Natural Science Foundation of China (NSFC) under grants (No. 81873099), National Science and Technology Major Project of the Ministry of Science and Technology of China (No. 2018ZX09735005), and New Teacher Start-up Fund of Beijing University of Chinese Medicine (No.2020-JYBXJSJJ-009).

\section{References}

[1] H. M. Gao, J. X. Liu, Z. M. Wang, and W. H. Wang, "Phytolacacinoside A, a new triterpenoid saponin from Phytolacca acinosa Roxb," Journal of Asian Natural Products Research, vol. 11, no. 5, pp. 433-438, 2009.

[2] G. Y. Yifei Li, "Review on pharmacological effects and toxicity of Phytolaccae," Chinese Journal of Experimental Traditional Medical Formulae, vol. 17, no. 13, pp. 248-251, 2020.

[3] J. Chen, "Clinical analysis on 26 cases of integrated traditional Chinese and western medicine in treating aeate Phytolacca acinosa poisoning," Journal of Zhejiang University of Traditional Chinese Medicine, vol. 34, no. 2, p. 221, 2019.
[4] G. Y. Qian Zhou, R. Jin, and J. Xie, "Study on renal cell toxicity induced by esculentoside A," World Chinese Medicine, vol. 9, no. 2, pp. 151-154, 2020.

[5] G. Y. Qian Zhang, R. Jin, and G. Cui, Preliminary Study on the Liver and Kidney Toxicity of Esculentoside A, p. 223, China Academic Journal Electronic Publishing House, Beijing, China, 2021.

[6] Z. Jia, C. Zhao, and M. Wang, "Hepatotoxicity assessment of Rhizoma Paridis in adult zebrafish through proteomes and metabolome," Biomedecine \& Pharmacotherapie, vol. 121, Article ID 109558, 2020.

[7] C. Zhao, Z. Jia, and E. Li, "Hepatotoxicity evaluation of Euphorbia kansui on zebrafish larvae in vivo," Phytomedicine: International Journal of Phytotherapy and Phytopharmacology, vol. 62, Article ID 152959, 2019.

[8] Y. Zhang, Y. Zhang, and J. Li, "The role of hepatic antioxidant capacity and hepatobiliary transporter in liver injury induced by isopsoralen in zebrafish larvae," Human \& Experimental Toxicology, p. 960327118774873, 2019.

[9] C. Zhao, M. Wang, and Z. Jia, "Similar hepatotoxicity response induced by Rhizoma Paridis in zebrafish larvae, cell and rat," Journal of Ethnopharmacology, vol. 250, Article ID 112440, 2020.

[10] C. Gomez-Canela, D. Tornero-Canadas, E. Prats, B. Pina, R. Tauler, and D. Raldua, "Comprehensive characterization of neurochemicals in three zebrafish chemical models of human acute organophosphorus poisoning using liquid chromatography-tandem mass spectrometry," Analytical and Bioanalytical Chemistry, vol. 410, no. 6, pp. 1735-1748, 2018.

[11] J. V. Goldstone, A. G. McArthur, and A. Kubota, "Identification and developmental expression of the full complement of Cytochrome P450 genes in Zebrafish," BMC Genomics, vol. 11, p. 643, 2010.

[12] W. Alderton, S. Berghmans, and P. Butler, "Accumulation and metabolism of drugs and CYP probe substrates in zebrafish larvae," Xenobiotica, vol. 40, no. 8, pp. 547-557, 2010.

[13] B. Wu, S. Liu, and X. Guo, "Responses of mouse liver to dechlorane plus exposure by integrative transcriptomic and metabonomic studies," Environmental Science Technology, vol. 46, no. 19, pp. 10758-10764, 2012.

[14] S. Y. Wu, N. N. Phan, and S. H. Ho, "Metabolomic assessment of arsenite toxicity and novel biomarker discovery in early development of zebrafish embryos," Toxicology Letter, vol. 290, pp. 116-122, 2018.

[15] C. Liu, C. Zhang, and W. Wang, "Integrated metabolomics and network toxicology to reveal molecular mechanism of celastrol induced cardiotoxicity," Toxicology and Applied Pharmacology, vol. 383, Article ID 114785, 2019.

[16] F. Cao, X. Liu, C. Wang, M. Zheng, X. Li, and L. Qiu, “Acute and short-term developmental toxicity of cyhalofop-butyl to zebrafish (Danio rerio)," Environmental Science and Pollution Research, vol. 23, no. 10, pp. 10080-10089, 2016.

[17] J. Ru, P. Li, and J. Wang, "TCMSP: a database of systems pharmacology for drug discovery from herbal medicines," Journal of Cheminformatics, vol. 6, p. 13, 2014.

[18] P. Zhang, H. Chen, and G. Shen, "Network pharmacology integrated with experimental validation reveals the regulatory mechanism of plastrum testudinis in treating senile osteoporosis," Journal of Ethnopharmacology, vol. 276, Article ID 114198, 2021.

[19] F. Liu, L. Li, J. Chen, Y. Wu, Y. Cao, and P. Zhong, “A network pharmacology to explore the mechanism of in the treatment of ischemic stroke," BioMed Research International, vol. 2021, Article ID 6611018, 20 pages, 2021. 
[20] G. Dennis, B. T. Sherman, and D. A. Hosack, "DAVID: database for annotation, visualization, and integrated discovery," Genome Biology, vol. 4, no. 5, p. P3, 2003.

[21] Q. Zeng, L. Li, and Y. Jin, "A network pharmacology approach to reveal the underlying mechanisms of pall. On the treatment of alzheimer's disease," Evidence-based Complementary and Alternative Medicine, vol. 2019, Article ID 8706589, 12 pages, 2019.

[22] M. I. Avigan, R. P. Mozersky, and L. B. Seeff, "Scientific and regulatory perspectives in herbal and dietary supplement associated hepatotoxicity in the United States," International Journal of Molecular Sciences, vol. 17, no. 3, p. 331, 2016.

[23] R. Nissan, A. Poperno, and G. Y. Stein, "A case of hepatotoxicity induced by adulterated "tiger king", a Chinese herbal medicine containing sildenafil," Current Drug Safety, vol. 11, no. 2, 2016.

[24] M. Wagner, G. Zollner, and M. Trauner, "New molecular insights into the mechanisms of cholestasis," Journal of Hepatology, vol. 51, no. 3, pp. 565-580, 2009.

[25] N. Anderson and J. Borlak, "Molecular mechanisms and therapeutic targets in steatosis and steatohepatitis," Pharmacology Review, vol. 60, no. 3, pp. 311-357, 2008.

[26] J. Li, Z. Zhao, J. Feng, J. Gao, and Z. Chen, "Understanding the metabolic fate and assessing the biosafety of $\mathrm{MnO}$ nanoparticles by metabonomic analysis," Nanotechnology, vol. 24, no. 45, Article ID 455102, 2013.

[27] J. Ma, M. Li, and P. K. Kalavagunta, "Protective effects of cichoric acid on $\mathrm{H} 2 \mathrm{O} 2$-induced oxidative injury in hepatocytes and larval zebrafish models," Biomedicine \& Pharmacotherapy, vol. 104, pp. 679-685, 2018.

[28] Y. X. Pan, Z. Luo, M. Q. Zhuo, C. C. Wei, G. H. Chen, and Y. F. Song, "Oxidative stress and mitochondrial dysfunction mediated Cd-induced hepatic lipid accumulation in zebrafish Danio rerio," Aquatic Toxicology, vol. 199, pp. 12-20, 2018.

[29] J. M. Mato, M. L. Martínez-Chantar, and S. C. Lu, "Methionine metabolism and liver disease," Annual Review of $\mathrm{Nu}$ trition, vol. 28, pp. 273-293, 2008.

[30] H. J. Wang, M. Zhang, W. J. Li, Y. Ni, J. Lin, and Z. H. Zhang, "An efficient $\mathrm{Ni} / \mathrm{Pd}$ catalyzed chemoselective synthesis of 1,3,2-benzodiazaborininones from boronic acids and anthranilamides," Advanced Synthesis \& Catalysis, vol. 361, p. 21, 2019.

[31] N. Krishnan, M. B. Dickman, and D. F. Becker, "Proline modulates the intracellular redox environment and protects mammalian cells against oxidative stress," Free Radical Biology \& Medicine, vol. 44, no. 4, pp. 671-681, 2008.

[32] S. K. Natarajan, W. Zhu, and X. Liang, "Proline dehydrogenase is essential for proline protection against hydrogen peroxide-induced cell death," Free Radical Biology \& Medicine, vol. 53, no. 5, pp. 1181-1191, 2012.

[33] B. Daignan-Fornier and B. Pinson, "Yeast to study human purine metabolism diseases," Cells, vol. 81 page, 2019.

[34] J. J. Moreno, "Effect of olive oil minor components on oxidative stress and arachidonic acid mobilization and metabolism by macrophages RAW 264.7," Free Radical Biology \& Medicine, vol. 35, no. 9, pp. 1073-1081, 2003. 